\title{
Comparison of Radiography, Ultrasonography and SNAP cPL in the Diagnosis of Acute Pancreatitis in Dogs
}

\author{
C.M. Abilaasha ${ }^{1}$, D. Chandrasekaran ${ }^{2 *}$, S. Kavitha ${ }^{1}$ and S. Vairamuthu ${ }^{3}$ \\ ${ }^{1}$ Department of Veterinary Clinical Medicine, Madras Veterinary College, Tamil Nadu Veterinary and Animal Sciences \\ University, Chennai, Tamil Nadu, INDIA \\ ${ }^{2}$ Department of Clinics, Madras Veterinary College, Tamil Nadu Veterinary and Animal Sciences University, Chennai, \\ Tamil Nadu, INDIA \\ ${ }^{3}$ Centralized Clinical Laboratory, Madras Veterinary College, Tamil Nadu Veterinary and Animal Sciences University, Chennai, \\ Tamil Nadu, INDIA \\ *Corresponding author: D Chandrasekaran; E-mail:drchandrus73@gmail.com
}

Received: 24 Sept., 2019

Revised: 20 Dec., 2019

Accepted: 02 Jan., 2020

\begin{abstract}
The study was aimed to compare various diagnostic modalities in the diagnosis of acute pancreatitis in dogs. Forty dogs with history of acute onset of vomiting, abdominal pain and anorexia were screened for acute pancreatitis by radiography, abdominal ultrasound and confirmed by SNAP cPL (Canine pancreatic lipase) kit test. Fourteen ( 0.375 per cent of gastrointestinal cases) dogs found to be positive for acute pancreatitis by abdominal ultrasound were selected and further subjected to radiography to rule out the underlying causes of acute pancreatitis and confirmed by SNAP cPL test. Out of fourteen dogs, four dogs (29 per cent) having specific radiographic changes gas filled intestinal loops with loss of serosal details, SNAP cPL was positive in nine dogs (64 per cent). Abdominal ultrasonography revealed hypoechoic pancreas, hyperechoic surrounding fat saponification, enlarged or irregular pancreas and dilatation of biliary or pancreatic duct as the major changes in acute pancreatitis dogs. Ultrasound was found to be superior to radiography in the diagnosis of acute pancreatitis and SNAP cPL was found to be effective in confirming only in acute stages of pancreatitis.
\end{abstract}

Keywords: Acute pancreatitis, Dogs, Radiography, Ultrasound, SNAP cPL

Acute pancreatitis is defined as sudden onset and severe inflammatory condition of pancreas, which is characterized by auto-digestion and necrosis of pancreas. Among all the exocrine pancreatic diseases, acute pancreatitis is considered as one of the commonly encountered condition in dogs (Mansfield et al., 2012).

Diagnosis of acute pancreatitis till date is considered as one of the most challenging disease among all gastrointestinal disorders. Dogs presented to the veterinarian with acute abdominal disease require timely intervention and accurate diagnosis for better survival rate (Thompson et al., 2009). Diagnosis of acute pancreatitis is based on array of tests, as no single test is considered to be specific for acute pancreatitis. Dogs are usually presented with history of anorexia, vomiting, cranial abdominal pain and lethargy and on physical examination tachycardia, tachypnea, dehydration, hypoglycemia and hypotension was clinically observed.

Sensitivity of radiography is recorded to be about 24 per cent in diagnosis of acute pancreatitis. Radiographical abnormalities revealed loss of cranial abdominal details, gas filled intestinal loops and other concurrent diseases had changes like neoplasia, foreign body and organomegaly (Andrew, 2013). Ultrasonography is considered as a better and non-invasive technique in early diagnosis of acute pancreatitis with sensitivity of about 63 per cent in dogs.

How to cite this article: Abilaasha, C.M., Chandrasekaran, D., Kavitha, S and Vairamuthu, S. (2020). Comparison of radiography, ultrasonography and SNAP CPL in the diagnosis of acute pancreatitis in dogs. J. Anim. Res. 10(1): 41-45. 
Major findings included enlarged, hypoechoic pancreas with hyperechoic surrounding peripancreatic fat (Nyland et al., 2015).

SNAP cPL has been found to be the most sensitive and specific test in the diagnosis of acute pancreatitis in dogs. It specifically measures canine pancreatic lipase, which is elevated during any inflammation of pancreas. It helps to rule out other acute abdominal diseases (Steiner, 2010). A better diagnostic aid for acute pancreatitis among various diagnostic modalities helps in timely diagnosis and medical management in dogs. The present study with the objective to compare various diagnostic modalities in the diagnosis of acute pancreatitis in dogs.

\section{MATERIALS AND METHODS}

Dogs with history of sudden onset of vomiting, abdominal pain, anorexia and lethargy presented to the Small Animal Out-Patient Medicine Unit of Madras Veterinary College Teaching Hospital, TANUVAS were screened for acute pancreatitis by abdominal ultrasound, radiography and confirmed by SNAP cPL kit test.

Selected clinical cases were subjected to radiographic examination to rule out other gastrointestinal disease or neoplasia (Mix and Jones, 2006) with Digital X-ray (AGFA, CR-30 X) as per standard procedure Brant and Helms (2012).

Selected clinical cases were subjected to ultrasonographic examination for confirmation of changes pertaining to pancreas and to differentiate it from other pancreatic disorders (Silke and George, 2007). Dogs were prepared by clipping the hair around the abdominal area for ultrasonographic examination. The right $9^{\text {th }}$ to $12^{\text {th }}$ intercostal space facilitates transverse views when excessive bowel gas was present. Positioning was done in dorsal recumbency, ventral recumbency, and lateral recumbency or in the standing posture. Ultrasound examination was done with ultrasound scanner (ESOATE, Mylab 20) using 5- 7.5 MHz high frequency probe as per standard procedure described by Nyland and Mattoon (2015).

Clinical cases showing pancreatic changes in ultrasound were subjected to SNAP cPL estimation for confirmation. Small quantity of serum sample was transferred separately in an eppendorf tube. Three drops of sample and 4 drops of conjugate were dispensed in a sample disposable tube and the sample tube was gently inverted for about 4-5 times to mix thoroughly. The entire contents was poured into the sample well of the SNAP device. Color appeared first in the activation circle, and the device was pressed firmly to activate. The results were recorded after 10 minutes (Steiner et al., 2009).

\section{RESULTS AND DISCUSSION}

Forty dogs suspected for acute pancreatitis were subjected to radiography and abdominal ultrasound examination for pancreatic changes using standard protocol. Fourteen dogs had typical ultrasonographic changes of acute pancreatitis and out of which four dogs (29 per cent) had specific radiographic findings and nine dogs (64 per cent) were confirmed for acute pancreatitis by SNAP cPL kit test.

The common radiographic findings in dogs with acute pancreatitis were gas filled intestinal loops with loss of serosal details (29 per cent), splenomegaly ( 22 per cent), normal study (21 per cent), gastro-intestinal foreign body (14 per cent), cardiomegaly ( 7 per cent) and hepatic mass (7 per cent).

Jennifer et al. (2003) documented radiographic findings of acute pancreatitis in dogs to be comprising mainly of loss of serosal details in the cranial abdomen, displacement of abdominal organs and gas filled intestinal loops. The present study is in full agreement with the above said authors as 29 per cent dogs were found to be presented with similar radiographic changes characterized by gas filled intestinal loops with loss of serosal details. The radiographic changes (71 per cent) in the present study are not specific in diagnosis of acute pancreatitis in dogs; however, it is considered as a very important, non-invasive diagnostic aid in ruling out other gastrointestinal disorders like gastrointestinal foreign bodies, free gas in abdomen, organ enlargement, neoplasia and fluid filled uterus (Saima et al., 2017).

Ultrasonographic findings in dogs with acute pancreatitis were hypoechoic pancreas (100 per cent), hyperechoic surrounding fat sponification (42.85 per cent) (Fig. 3), enlarged or irregular pancreas (50 per cent) (Fig. 2) and dilatation of biliary or pancreatic duct (14.29 per cent).

In the present study, decreased echogenicity of pancreas may be due to edema, necrosis or hemorrhage, and 
inflammatory exudates secondary to pancreatitis (Chee and Steiner, 2016). In the present study, enlarged hypoechoic pancreas with hyperechoic mesentery was clearly visualized which might be due to necrosis of peripancreatic fat, consistent with saponification of fat secondary to inflammation and concurred with the findings of Hecht and Henry, (2007). Dilatation of the pancreatic duct was observed in pancreatitis which might be due to intramural edema and dilatation or obstruction of the bile ducts (Avante et al., 2018).

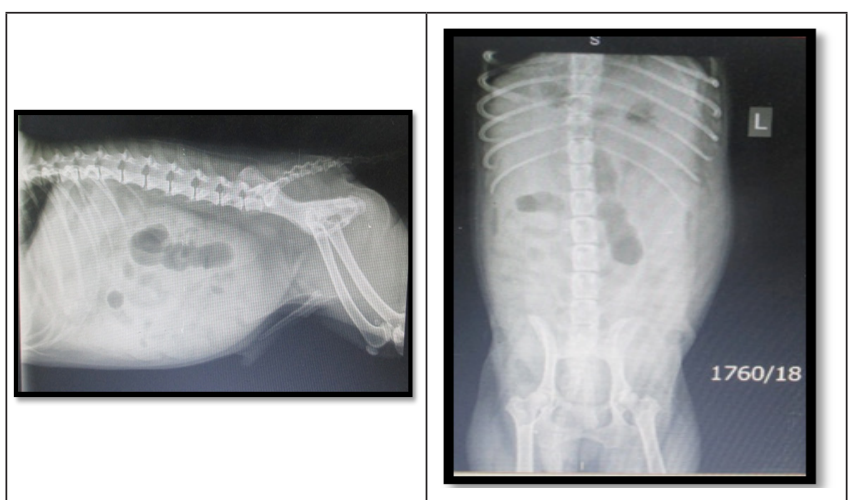

Fig. 1: Radiography - Lateral and Ventro-dorsal view of abdomen showing gas filled intestinal loops with ground glass appearance in acute pancreatitis in dogs

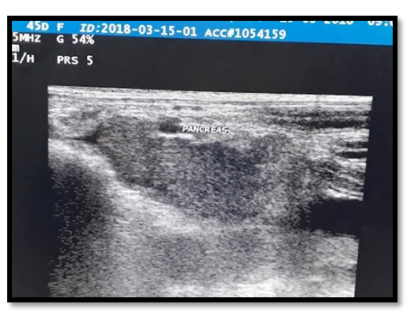

Fig. 2: Ultrasonographic changes in acute pancreatitisenlarged and hypoechoic pancreas

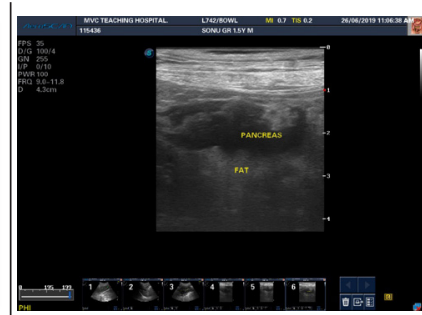

Fig. 3: Hypoechoic, diffused pancreas with hyperechoic fat surrounding pancreas
In the present study, nine dogs (64 per cent) were found to be positive for SNAP cPL kit test (Fig. 4A) and five were negative (36 per cent) (Fig. 4B).

The SNAP cPL has 96 to 100 per cent agreement with the Spec cPL in the samples with normal pepsin like immunoreactivity (PLI) and 88 to 92 per cent agreement in samples with PLI. Further indicating that, in cases of mild to moderate index of suspicion of pancreatic damage, a positive SNAP cPL result should be confirmed with quantitative Spec cPL (Beall et al., 2011).

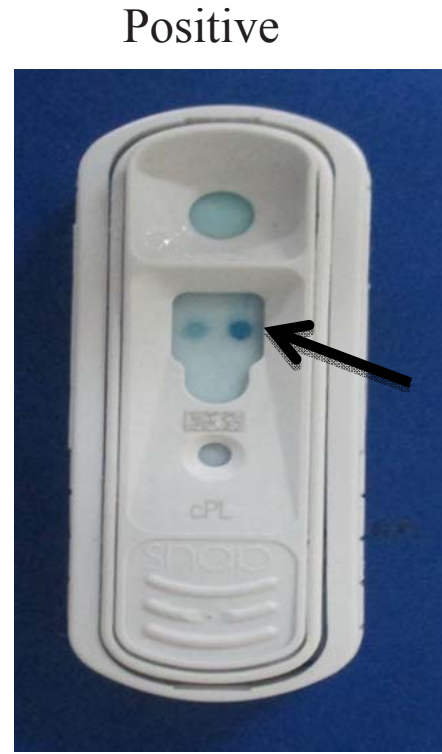

(A)

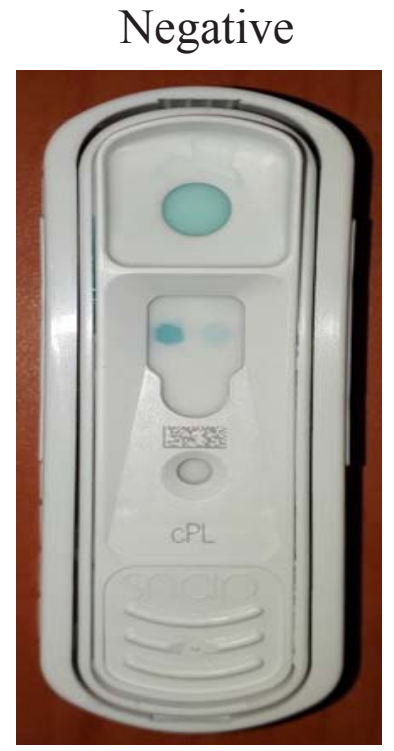

(B)
Fig. 4: Diagnostic test results on the basis of development of color for acute pancreatitis in dog. (A) SNAP cPL kit: Positive for acute pancreatitis in a dog; (B) SNAP cPL kit: Negative for acute pancreatitis suspected dog

Cridge et al. (2018) concluded that, no single assay had high enough diagnostic specificity to conclusively diagnose pancreatitis. A combination of signalment, physical examination, heaematological and serum biochemical analysis, pancreatic enzyme elevation, abdominal ultrasound examination and SNAP cPL estimation, may be the most practical means of establishing a definitive diagnosis of clinical pancreatitis in dog.

In the present study, there was a significant difference of 6.09 chi square value upon comparison of accuracy of ultrasound and SNAP cPL (Fig. 5 and Table 1) for diagnosis of acute pancreatitis in dogs.

Table 1: Comparison of diagnostic modalities of acute pancreatitis dogs

\begin{tabular}{ll}
\hline Parameters & Chi- square Test \\
\hline Ultrasound \& SNAP cPL & $6.09^{*}$ \\
Ultrasound \& Radiography & $13.29^{* *}$ \\
\hline
\end{tabular}


This is in agreement with the observations made by Mansfield et al. (2000) who stated that, improved technology and training has no doubt improved the sensitivity of ultrasound as a diagnostic modality from previously published rates of approximately 66 per cent, but the true sensitivity is unknown and is likely to be highly operator and equipment dependent. Poor visualization of the pancreas due to gas interference from the gastrointestinal tract can hamper proper diagnosis (Nyland et al., 2015). Steiner (2008) concluded that, abdominal ultrasonography also appeared to be useful in diagnosing pancreatitis but may be more sensitive in dogs with severe pancreatic pathology.

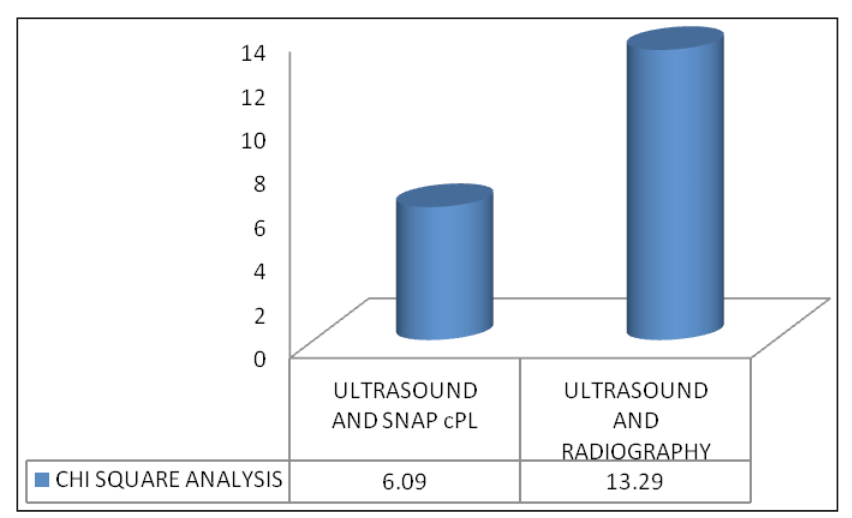

Fig. 5: Comparison of different diagnostic modalities of acute pancreatitis in dogs $(n=14)$.

Steiner (2010) stated that, a negative SNAP cPL helps the clinician to quickly shift the focus of the clinical investigations to other conditions. In the present study, out of all the fourteen cases selected having pancreatic changes in ultrasound, SNAP cPL was positive in nine dogs. The reason might be delayed presentation of the case to the hospital ( $>90 \mathrm{~min}$ ) in case of secondary pancreatitis, where a decrease in the ongoing inflammatory process of pancreas resulting in a negative SNAP. Thus the decrease in the quantity of canine pancreatic lipase levels below the detectable limit helps in judging the severity of acute pancreatitis up to a certain level in case of secondary pancreatitis due to concurrent diseases.

In the present study, there was a highly significant difference of 13.29 chi square value upon comparison of accuracy of ultrasound and radiography (Fig. 5 and Table 1) for diagnosis of acute pancreatitis in dogs.
In the present study, only 29 per cent of cases showed radiographic changes such as gas filled intestinal loops and loss of serosal details suggestive of secondary changes as sequel of pancreatitis, whereas changes in the remaining cases were not indicative of pancreatitis. Thus, indicating less sensitivity and specificity of radiography when compared to ultrasound in the diagnosis of acute pancreatitis in dogs. The results of the present study are in agreement with Mansfield et al. (2011) who stated that, abdominal radiographs, generally are not useful for the diagnosis of pancreatitis, yet essential to rule out surgical conditions such as pyometra or intestinal foreign body obstruction and should not be neglected or overlooked in general practice.

Therefore, ultrasonography was found to be superior to radiography in the diagnosis of acute pancreatitis in dogs. This finding is in agreement with Graham (2006), who stated that ultrasonography has a better sensitivity of $\sim 70$ per cent compared to radiography in diagnosis of acute pancreatitis in dogs.

\section{CONCLUSION}

Forty dogs with history of acute onset of vomiting, abdominal pain and anorexia were screened for acute pancreatitis by radiography, abdominal ultrasound and confirmed by SNAP cPL kit test. Fourteen $(0.375$ per cent of gastrointestinal cases) dogs found to be positive for acute pancreatitis by abdominal ultrasound, out of which four dogs (29 per cent) having specific radiographic changes gas filled intestinal loops with loss of serosal details and SNAP cPL was positive in nine dogs (64 per cent). Abdominal ultrasonography revealed hypoechoic, irregular pancreas. Hyper echoic fat saponification and dilatation of biliary or pancreatic duct as the changes typical of acute pancreatitis. Ultrasound was found to be superior to radiography in the diagnosis of acute pancreatitis and SNAP cPL was found to be effective in confirming only in acute stages of pancreatitis.

\section{REFERENCES}

Andrew, L. 2013. Canine pancreatitis. Emergency Medicine/ Gastroenterology/ Hepatology, pp. 83-86.

Avante, M.L., Silva, P.D.A.D., Feliciano, M.A.R., Maronezi, M.C., Simoes, A.R., Uscategui, R.A.R. and Canola, J.C. 
2018. Ultrasonography of the canine pancreas. Rev. $M V Z$ Córdoba., 23(1): 6552-6563.

Beall, M.J., Cahill, R., Pigeon, K., Hanscom, J. and Huth, S.P. 2011. Performance validation and method comparison of an in-clinic enzyme-linked immunosorbent assay for the detection of canine pancreatic lipase. J. Vet. Diagn. Invest., 23: 115-119.

Brant, W.E. and Helms, C.A. 2012. In: Fundamentals of Diagnostic Radiology, $7^{\text {th }}$ edition. Wolters Kluwer/Lippincott Williams and Wilkins Health, Philadelphia, pp. 792.

Chee, H.C. and Steiner, J.M. 2016. From diagnosis to treatment - A case of canine acute pancreatitis. ACVIM \& ECVIM. pp. 52-59.

Cridge, H., MacLeod, A.G., Pachtinger,G.E., Mackin,A.J., Sullivant,A.M., Thomason, J.M., Archer, T.M., Lunsford, K.V., Rosenthal,K. and Wills, R.W. 2018. Evaluation of SNAP cPL, Spec cPL, VetScan cPL rapid test, and precision PSL assays for the diagnosis of clinical pancreatitis in dogs. J. Vet. Intern. Med., 32: 658-664.

Graham, S. 2006. Canine Pancreatitis. IDEXX Laboratories.

Hecht, S. and Henry, G. 2007. Sonographic evaluation of the normal and abnormal pancreas. Clin. Tech. Small Anim. Pract., 22: 115-121.

Jennifer, L.H., Daniel, L.C. and Elizabeth, A.R. 2003. Acute pancreatitis in dogs. J. Vet. Emerg. Crit. Care ., 13(4): 201 -213 .

Mansfield, C.S and Jones, B.R. 2000. Plasma and urinary trypsinogen activation peptide in healthy dogs, dogs with pancreatitis and dogs with other systemic diseases. Aust. Vet. $J ., 78(6)$ : 416-422.

Mansfield, C.S., Watson, P.D. and Jones, B.R. 2011. Specificity and sensitivity of serum canine pancreatic elastase-1 concentration in the diagnosis of pancreatitis. J. Vet. Diagn. Invest., 23(4): 691-697.
Mansfield, C.S., Anderson, G.A. and O'Hara, A.J. 2012. Association between canine pancreatic-specific lipase and histologic exocrine pancreatic inflammation in dogs assessing specificity. J. Vet. Diagn. Invest., 24(2): 312-318.

Nyland, T.G. and Mattoon, J.S.2015. Chapter: II Pancreas. Small animal diagnostic ultrasound, $3^{\text {rd }}$ edition, pp: 438-467.

Saima, M., Farooq, I., Rashid, S.M., Rehman, M.U., Ali, R., Shabir, M., Mir, M.U.R. and Ahmad, S.B. 2017. Acute pancreatitis in dogs: A review. Pharm. Innov. J., 6(12): 509516.

Silke, H and George, H. 2007. Sonographic evaluation of the normal and abnormal pancreas. Clin. Tech. Small Anim. Pract., 22: 115-121.

Steiner, J.M., Newman, S.J., Xenoulis, K., Woosley, K., Suchodolski, J., Williams, D. and Barton, L. 2008. Sensitivity of serum markers for pancreatitis in dogs with macroscopic evidence of pancreatitis. Vet. Ther., 9(4): 263-273.

Steiner, J., Teague, S.R., Lees, G.E., Willard, M.W., Williams, D.A. and Ruaux, C.G. 2009. Stability of canine pancreatic lipase immunoreactivity concentration in serum samples and effects of long term administration of prednisone to dogs on serum canine pancreatic lipase immunoreactivity concentrations. Am. J. Vet. Res., 70(8): 1001-1005.

Steiner, J.M. 2010. New tests for Pancreatitis. NAVC Clinician's Brief, pp. 75-78.

Thompson, L.J., Seshadri, R. and Raffe, M.R. 2009. Characteristics and outcomes in surgical management of severe acute pancreatitis: 37 dogs (2001-2007). J. Vet. Emerg. Crit. Care., 19(2): 165-173. 
